\title{
DIVING INTO MINORITY STRESS THEORY WITHIN THE LGBTQ+ COMMUNITY
}

\author{
Sydney Downing \\ Human Development Family Studies, Bowling Green State University (USA)
}

\begin{abstract}
The LGBTQ+ (Lesbian, Gay, Bisexual, Transgender, Queer) community is an evolving population with a variety of identities that need to be understood by the heteronormative society. The first step for understanding this community is for educators to grasp current terminology and concepts. Moreover, the heteronormative society has impacted individuals in the LGBTQ+ society due to socially constructed ideals of identity. Specifically, the intersection of race, gender, and religion in the LGBTQ+ community relating to sexual violence and the like. The purpose of this presentation is to convey the need for sex education that includes the LGBTQ+ community, sexual orientation, gender identities, and gender expression. A second purpose is to investigate the impact on individuals of the LBGTQ+ populations based on the Minority Stress Theory. Minority Stress Theory breaks down the amount of stress a person experiences due their minority status using the minority stress model. As well as, present a call for action for educators at all levels. It is important to deliver and to have professional development centered around Safe Zone Trainings, which is a training the educates on the identities within LGBTQ+ communities and how as a society we can be better allies. Lastly, synthesize information about the LGBTQ+ individual's experiences, future directions and research that needs to be completed.
\end{abstract}

Keywords: Heteronormative, minority stress theory, safe zone, $L G B T Q+$, sex education.

\section{Introduction and current problem}

Heteronormativity is an ideology that promotes gender conventionality, heterosexuality, and family traditionalism as the correct way for people to be (Oswald, Blume, \& Marks, 2018). It is a framework that surrounds the practices of heterosexuality and because heterosexuality is "more natural" than other forms of sexuality it should be standard sexual practice (Oswald et. al., 2018). It perpetrates heterosexual privilege which are advantages that come with heterosexuality in this society and culture (i.e. acceptance from family, safety, and acceptance in their chosen career field). As well as, heterosexism, which is the belief that all people are heterosexual, the assumption and/ or belief that heterosexual relationships and behavior are superior to other forms of relationships. The term queering processes relates to acts and ideas that defy heteronormativity by challenging gender, sexuality and/or family binaries (Oswald et. al., 2018). Gender identity refers to how a person perceives themselves and what they call themselves; this may or may not agree with the societal gender roles outlined for their sex which are typically male and female. Gender roles are the societal and cultural expectations of people based upon their biological sex usually masculine/feminine. Butler (1990) argues that gender is socially constructed, and gender expression is a social performance. The binary divide (male and female) is also socially created. Acceptable and valued behaviors and roles are defined and reinforced through culture and media. Queer theory assumes this binary divide is not fixed and "to queer" is to challenge social constructs. The goal of this literature review is to analyze how heteronormativity impacts sexual minority populations through the lenses of minority stress theory, sexual violence, and intersectionality; and propose a resolution for change in academia settings.

\section{Minority stress theory}

Minority Stress Theory breaks down the amount of stress a person experiences due their minority status using the minority stress model. Minority stress considers the additional stress individuals from stigmatized social categories experience as a result of their minority position (Meyer, 2013). The minority stress model (See Figure 1) describes stress processes, such as the experience of prejudice events, expectations of rejection, hiding and concealing, internalized homophobia, and coping and social support (Meyer, 2013). Stressors are heighted when multiple intersectional identities are included. 
Figure 1. Minority Stress Model.

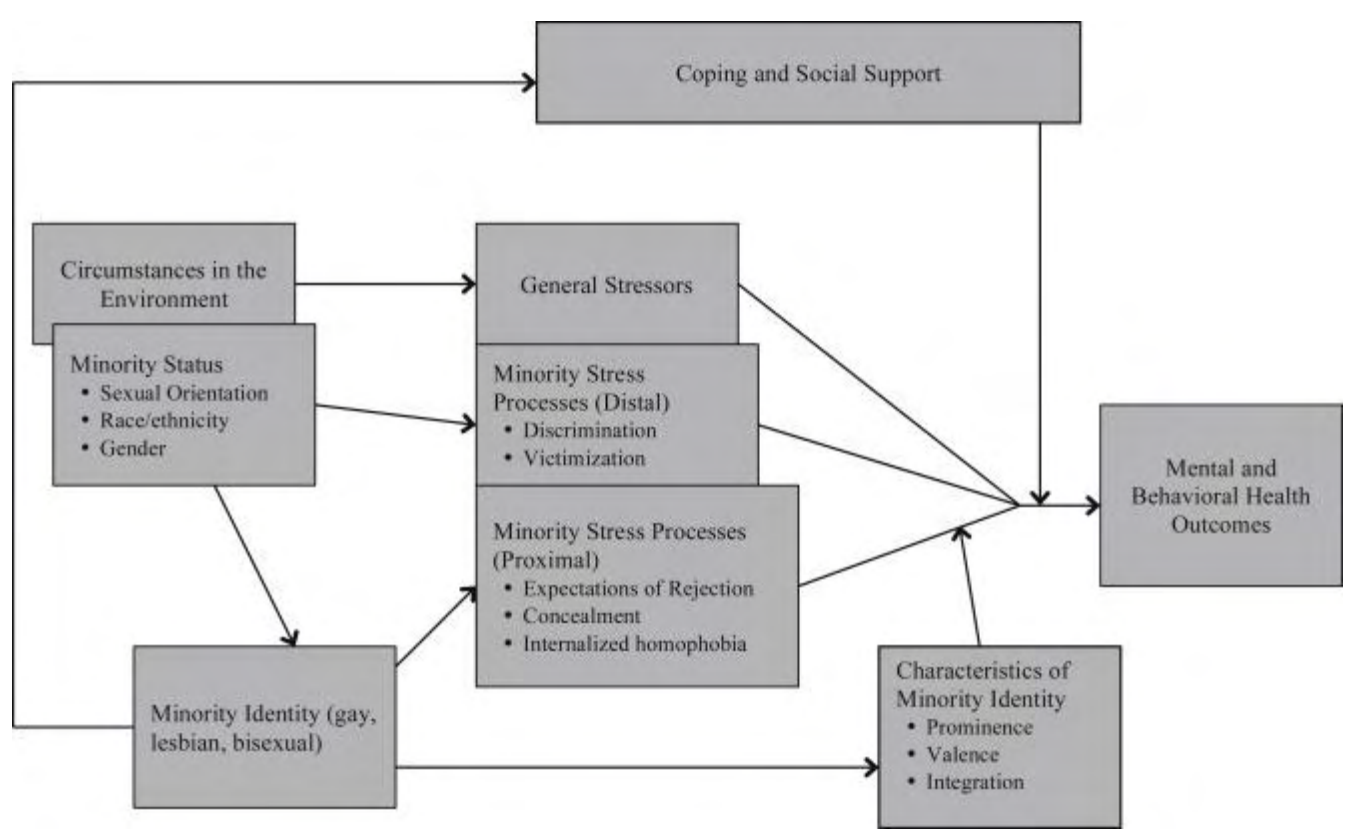

\subsection{Intersectionality and sexual violence}

Intersectionality refers to the categorical and hierarchical classifications such as race, class, gender, sexuality, and nationality (Cole, 2019). Intersectionality is not just about oppressions, it is also about privilege and how individuals' positions in society affect which side of social classifiers they have (See Figure 2). For example, a white gay man would have the minority stressors from being gay as white is the dominant or privileged identity. However, for a black gay woman the minority stress is more intense because not only is the sexual minority in play but it also factors in racism, and sexism on top of any identities that are already held.

Figure 2. Intersectionality Matrix.

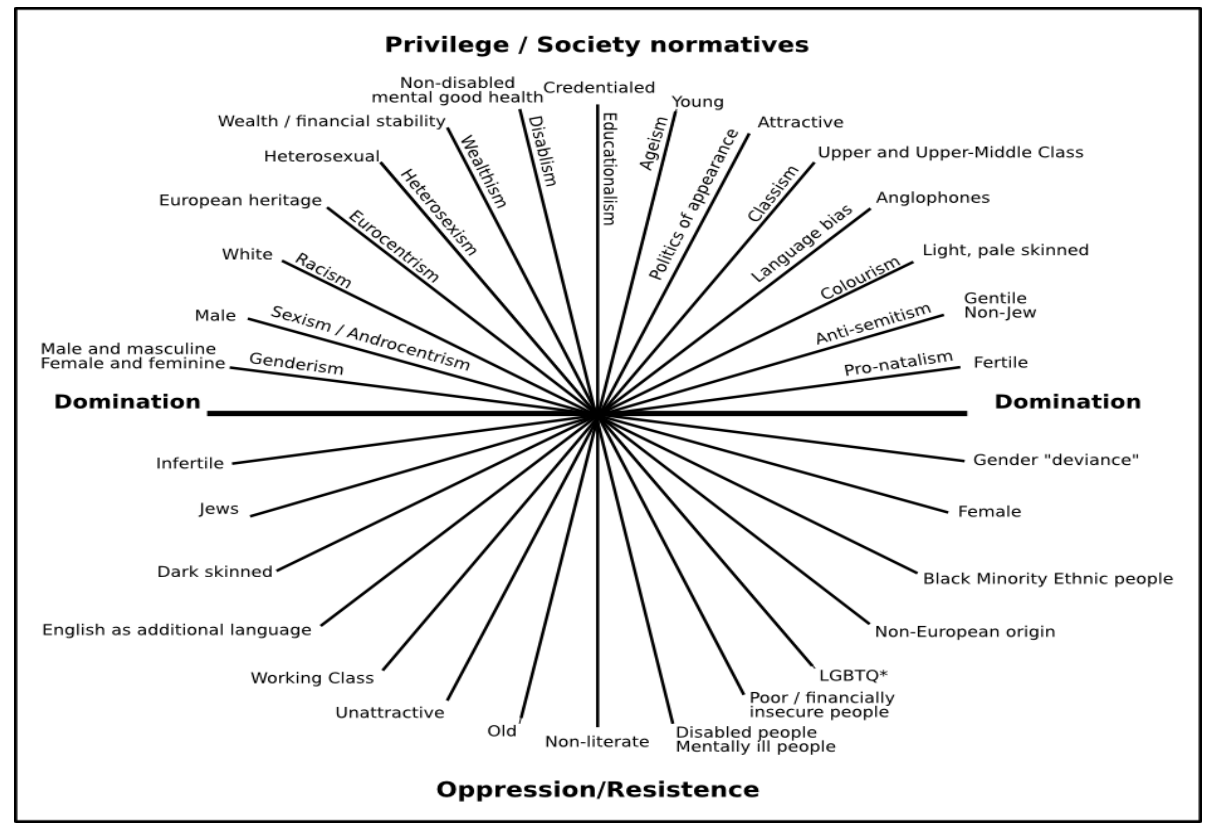

Little is known about intimate partner violence (IPV), sexual violence (SV), and stalking among the LGBTQ+ in the United States (Center for Disease Control and Prevention, 2010). "LGBTQ+ individuals are often targeted for sexual violence based on their sexual orientation and gender identities. In this way, sexual violence is used as a form of power, oppression, and control," according to Macalester University. According to The National Intimate Partner and Sexual Violence Survey (2010) approximately 1 in 5 bisexual women (22\%) and nearly 1 in 10 heterosexual women (9\%) have been raped by an intimate partner in their lifetime. According to RAINN, " $21 \%$ of TGQN (transgender, 
genderqueer, nonconforming) college students have been sexually assaulted, compared to $18 \%$ of non-TGQN females, and 4\% of non-TGQN males" rates that are substantially higher than those who identify as heterosexual.

\section{Limitations and future research}

In the current literature review, a major limitation is the limited amount of research done in the LGBTQ+ community. The scope of the paper is also limited to the geographic location of the United States. The timeline of research used is fairly recent besides some older fundamental models and books. However, research and studies are still being done since research has not begun primarily until the past two decades. Further research in this community may have go outside of the scopes of the typical way of conducting research to get accurate and substantial data collections. As research is also engaging in dialogues to talk to folks in a community and not just those in the ivory tower of academia.

\section{Conclusion and resolution}

According to Kulick, Wernick, Woodford \& Renn (2016), “Oppressive systems can have deleterious effects on health and wellbeing of marginalized communities." Barriers to reporting within the LGBTQ+ community range from "access to sexual health education, crisis support services, and health care... to the way the heteronormativity, substance abuse, internalized homophobia, rape culture, and misogyny affect our communities" (LGBT Individuals and Sexual Violence, 2019). According to Kulick et. al., (2016) reports stated that "engaging in efforts specifically focusing on heterosexism can also have a direct impact on individual wellbeing thorough the development of confidence and self-efficacy among LGBTQ students." Moving forward, in regards to educational settings having competent, inclusive, and intersectional sex education compared to rarely having sex education could decrease some minority stress that those in the LGBTQ+ population face (Parenthood, 2019). Intersectional work aims to be more inclusive to all people and respect every part of an individual's identity. Employees can take an active role by asking colleagues and students their preferred pronouns. As departments, having professional development around the training "Safe Zone" which is a welcoming, supportive and safe environment for (LGBT) students could create safe and affirming environments wherever they go (Human Rights Campaign, 2019). Studies show that in schools with inclusive curriculum students were more likely to feel more connected, comfortable speaking about their identity, and have accurate information regarding LGBT folks. Minority stress, intersectionality, and sexual violence will always be a part of communities, however, finding ways to include LGBTQ+ curriculum into education and challenging social constructs can lessen the minority stress that LGBTQ+ folks encounter.

\section{References}

Butler, J. (1990). Gender trouble: Feminism and the subversion of identity. New York: Routledge, Taylor \& Francis Group.

Cole, N. L. (2019, January 28). Understanding Intersectionality and Why It Matters to Sociologists. Retrieved from https://www.thoughtco.com/intersectionality-definition-3026353

Human Rights Campaign. (2019). Establishing an Allies/Safe Zone Program. Retrieved from https://www.hrc.org/resources/edtablishing-an-allies-safe-zone-program.

Kulick, A., Wernick, L. J., Woodford, M. R., \& Renn, K. (2016). Heterosexism, Depression, and Campus Engagement Among LGBTQ College Students: Intersectional Differences and Opportunities for Healing. Journal of Homosexuality, 64(8), 1125-1141. Doi:10.1080/00918369.2016.1242333

LGBT Individuals and Sexual Violence. (2019). Retrieved from https://www.macalester.edu/violenceprevention/lgbt/

Meyer, I. H. (2013). Prejudice, social stress, and mental health in lesbian, gay, and bisexual populations: Conceptual issues and research evidence. Psychology of Sexual Orientation and Gender Diversity, 1(S), 3-26. Doi:10.1037/2329-0382.1.s.3

National Resources for Sexual Assault Survivors and their Loved Ones. (2019). Retrieved from https://www.rainn.org/national-resourcs-sexual-assault-survivors-and-their-loved-ones Oswald, R. F., Blume, L. B., \& Marks, S. R. (2018). Decentering Heteronormativity: A Model for Family Studies. Sourcebook of Family Theory and Research, 143-165. Doi:10:4135/9781412990172.n6

Parenthood. P. State of Sex Education in USA Health Education in Schools. (2019). Retrieved from https://www.plannedparenthoo.org/learn/for-educators/whats-state-sex-education-us

The National Intimate Partner and Sexual Violence Survey (NISVS) Violence

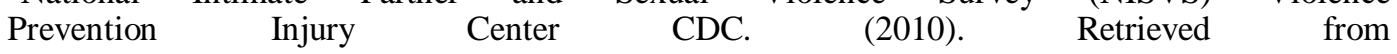
https://www.cdc.gov/violenceprevention/datasources/nisvs/index.html 